Publications of the Astronomical Society of the Pacific, 119: 398-406, 2007 April

(C) 2007. The Astronomical Society of the Pacific. All rights reserved. Printed in U.S.A.

\title{
Spectroscopic Determination of Radius Changes of Cepheid Variable Stars
}

\author{
David F. Gray and Kevin B. Stevenson \\ Department of Physics and Astronomy, University of Western Ontario, London, ON, Canada; dfgray@uwo.ca, ksteve24@uwo.ca \\ Received 2007 January 20; accepted 2007 March 15; published 2007 April 24
}

\begin{abstract}
We introduce a simple method for determining pulsation velocities of Cepheid variables; namely, the matching of observed spectral line profiles in both shape and absolute position with model profiles. This approach avoids the ambiguous meaning of radial velocity for asymmetric lines, and it avoids the awkward projection factor (the " $p$ " factor) used to expand radial velocities to pulsation velocities. In this investigation, we employ a simple pure-geometry model that reproduces all the major features in the observations. We illustrate the method using spectroscopic observations of $\zeta \mathrm{Gem}, \eta$ Aql, and $\delta$ Cep taken at the Elginfield Observatory, and derive the absolute radius changes.
\end{abstract}

\section{INTRODUCTION}

The radial pulsation of Cepheid variables is interesting on several fronts. The complex nature of the pulsations, their interactions with convection and granulation, and the differential rotation induced during the cycle pique our interest and offer difficult challenges to our understanding. Cepheids offer through their period changes one of the few ways to observe changes in stellar structure during our lifetime. In another arena, Cepheids are used to help set up the fundamental distance scale of the universe by combining the measured radius change in absolute units, obtained by integrating the radial velocity over a pulsation cycle, with the angular change that varies inversely with the distance. The angular radius and its changes can be measured photometrically by using magnitudes and color indices, as done in the original Baade-Wesselink method (Baade 1926; Wesselink 1946), or by fitting energy distributions (Gray 1967, 2005), or directly, by using interferometers (Kervella et al. 2001; Lane et al. 2002). In this investigation, we address only the determination of the radius change in kilometers.

Classical methods for determining the radius change start with observed radial velocities expressed as a function of pulsation cycle phase. Fortunately, the physical attributes of Cepheids seem to be reproducible cycle after cycle, so constructing the phase diagram presents no obstacle. Unfortunately, serious ambiguity occurs in the meaning of radial velocity, because the star's pulsation produces asymmetric line profiles. How does one define the Doppler shift of an asymmetric line? One can measure the position of the line core, the position midway between the continuum and the core, the position of the centroid, and so on. Differences can amount to several $\mathrm{km} \mathrm{s}^{-1}$. This question has been addressed in several publications (Kraft 1967; Wallerstein et al. 1992; Sasselov \& Lester 1990; Butler 1993; Kiss \& Vinkó 2000; and Nardetto et al. 2006, among others), and although various recipes have been suggested, the essential ambiguity remains. The method we present here completely sidesteps this issue by modeling the absolute position of the whole line, asymmetries included.

The next step in the classical method is to expand the observed radial velocities by a projection factor, typically $\sim 1.35$, to allow for the fact that the radial (on the star) pulsation velocities are only along our line of sight for the center of the stellar disk and decline to zero at the limb. The net Doppler shift in the observed spectra is therefore considerably smaller than the pulsation velocity. These projection factors range from 1.2 to 1.5 , depending on who computes them and what technique is used to measure the radial velocities. Furthermore, the projection factor varies with phase, although this detail is often ignored. More recent discussions and calculations of projection factors can be found in Hindsley \& Bell (1986), Sasselov \& Lester (1990), Sabbey et al. (1995), and Nardetto et al. (2004, 2006). The method we present here does not use projection factors. The pulsation velocity is a fundamental parameter of the models; when the computed profile matches the observations, we have the pulsation velocity directly, without the intermediary of a projection factor.

Another of our goals is to see how far simple geometry can be used to explain the line profiles and their changes. No radiation transfer is included. We are quite aware of many of the extensive hydrodynamical modeling efforts toward understanding Cepheid spectral lines and their variations, such as Karp (1975), Sasselov \& Karovska (1994), Sabbey et al. (1995), Fokin et al. (1996), Marengo et al. (2003), and Nardetto et al. (2004). None of these endeavors adequately include rotation and its likely variation with pulsation phase. Although hydrodynamical computations are certainly necessary for the understanding of how pulsations work, they can be involved and uncertain. Instead of pushing toward more complexity, we concentrate on the geometrical effects, and as we show here, such models turn out to be remarkably effective, although certainly 
Table 1

$\zeta$ GEMINORUM DATA

\begin{tabular}{|c|c|c|c|c|}
\hline $\begin{array}{c}\mathrm{JD}-2,440,000 \\
\text { (days) }\end{array}$ & Phase & $\begin{array}{c}V_{\text {puls }} \\
\left(\mathrm{km} \mathrm{s}^{-1}\right)\end{array}$ & $\begin{array}{c}v \sin i \\
\left(\mathrm{~km} \mathrm{~s}^{-1}\right)\end{array}$ & $\begin{array}{c}\zeta \\
\left(\mathrm{km} \mathrm{s}^{-1}\right)\end{array}$ \\
\hline $13,679.943$ & 0.770 & -7.0 & 8.0 & 10.0 \\
\hline $13,686.845 \ldots \ldots$ & 0.450 & 18.6 & 11.0 & 8.5 \\
\hline $13,762.676 \ldots \ldots$ & 0.922 & -9.7 & 8.0 & 8.8 \\
\hline $13,762.757$ & 0.930 & -10.0 & 8.0 & 8.5 \\
\hline $13,799.597 \ldots \ldots$ & 0.560 & 18.2 & 13.0 & 11.2 \\
\hline $13,810.596 \ldots \ldots$ & 0.640 & 7.1 & 10.0 & 11.8 \\
\hline $13,810.696 \ldots \ldots$ & 0.654 & 7.8 & 10.0 & 11.6 \\
\hline $13,812.612 \ldots \ldots$ & 0.843 & -8.3 & 8.0 & 9.0 \\
\hline $13,812.711 \ldots \ldots$ & 0.852 & -8.5 & 8.0 & 9.0 \\
\hline $13,815.612 \ldots \ldots$ & 0.138 & -13.0 & 8.0 & 7.8 \\
\hline $13,815.697 \ldots \ldots$ & 0.147 & -11.5 & 8.0 & 8.0 \\
\hline $13,821.590 \ldots \ldots$ & 0.727 & -4.8 & 8.0 & 11.0 \\
\hline $13,825.598$ & 0.120 & -14.6 & 8.0 & 8.0 \\
\hline $13,834.597 \ldots \ldots$ & 0.009 & -15.2 & 8.0 & 9.0 \\
\hline $13,841.597 \ldots \ldots$ & 0.699 & -1.4 & 8.0 & 11.8 \\
\hline $13,842.666 \ldots \ldots$ & 0.800 & -8.0 & 8.0 & 9.5 \\
\hline $13,844.608 \ldots \ldots$ & 0.996 & -14.2 & 8.0 & 9.2 \\
\hline $13,845.608 \ldots \ldots$ & 0.094 & -15.2 & 8.0 & 8.0 \\
\hline $13,860.596 \ldots \ldots$ & 0.572 & 17.8 & 12.0 & 11.0 \\
\hline
\end{tabular}

not perfect. Naturally, the geometrical model tells us very little about the physics of the pulsation.

Although the use of line profiles is a significant improvement over simple radial velocities in determining pulsation velocities, there is a cost. Our method requires considerably more observing time and reduction effort. The relatively high spectral resolution required with our approach means that it will be limited to the Cepheids brighter than $\sim 10$ th magnitude; the classical radial velocity technique will likely continue to be used for the fainter ones.

\section{OBSERVATIONS AND PROFILE SELECTION}

Three bright Cepheids were chosen to test our ideas: $\zeta$ Gem (HR 2650), $\eta$ Aql (HR 7570), and $\delta$ Cep (HR 8571). Tables 1,2 , and 3 summarize the observations, all taken at the Elginfield Observatory at the University of Western Ontario. The coudé spectrograph has been described elsewhere (e.g., Gray

Table 2

$\zeta$ Aquilae Data

\begin{tabular}{ccrcr}
\hline \hline $\begin{array}{c}\mathrm{JD}-2,440,000 \\
(\text { days })\end{array}$ & Phase & $\begin{array}{c}V_{\text {puls }} \\
\left(\mathrm{km} \mathrm{s}^{-1}\right)\end{array}$ & $\begin{array}{c}v \sin i \\
\left(\mathrm{~km} \mathrm{~s}^{-1}\right)\end{array}$ & $\begin{array}{c}\zeta \\
\left(\mathrm{km} \mathrm{s}^{-1}\right)\end{array}$ \\
\hline $53,662.548 \ldots \ldots$ & 0.319 & -9.0 & 10.0 & 10.0 \\
$53,686.503 \ldots \ldots$ & 0.657 & 14.0 & 13.0 & 8.8 \\
$53,878.837 \ldots \ldots$ & 0.456 & 0.0 & 10.0 & 11.0 \\
$53,892.836 \ldots \ldots$ & 0.407 & -3.0 & 10.0 & 10.0 \\
$53,902.784 \ldots \ldots$ & 0.793 & 30.3 & 15.0 & 10.0 \\
$53,917.707 \ldots \ldots$. & 0.873 & 25.0 & 15.5 & 13.0 \\
$53,922.791 \ldots \ldots$. & 0.580 & 5.2 & 10.0 & 10.5 \\
$53,923.722 \ldots \ldots$ & 0.710 & 21.5 & 13.0 & 9.0 \\
$53,930.743 \ldots \ldots$. & 0.688 & 18.3 & 13.0 & 8.8 \\
$53,932.741 \ldots \ldots$ & 0.967 & -12.8 & 10.0 & 12.8 \\
$53,933.746 \ldots \ldots$ & 0.108 & -21.7 & 10.0 & 9.5 \\
\hline
\end{tabular}

Table 3

$\delta$ Cephei Data

\begin{tabular}{|c|c|c|c|c|}
\hline $\begin{array}{c}\mathrm{JD}-2,440,000 \\
\text { (days) }\end{array}$ & Phase & $\begin{array}{c}V_{\text {puls }} \\
\left(\mathrm{km} \mathrm{s}^{-1}\right)\end{array}$ & $\begin{array}{c}v \sin i \\
\left(\mathrm{~km} \mathrm{~s}^{-1}\right)\end{array}$ & $\begin{array}{c}\zeta \\
\left(\mathrm{km} \mathrm{s}^{-1}\right)\end{array}$ \\
\hline $53,679.568$ & 0.470 & 8.5 & 9.0 & 7.0 \\
\hline $53,686.568 \ldots \ldots$ & 0.781 & 26.0 & 12.0 & 10.5 \\
\hline $53,686.695 \ldots \ldots$ & 0.798 & 24.0 & 12.5 & 10.5 \\
\hline $53,841.848 \ldots \ldots$ & 0.711 & 25.0 & 9.5 & 9.5 \\
\hline $53,842.850 \ldots \ldots$ & 0.899 & -15.8 & 10.0 & 9.3 \\
\hline $53,845.836 \ldots \ldots$ & 0.455 & 7.2 & 9.0 & 7.0 \\
\hline $53,878.742 \ldots \ldots$ & 0.587 & 15.0 & 10.5 & 8.0 \\
\hline $53,902.683 \ldots \ldots$ & 0.049 & -21.5 & 8.0 & 7.5 \\
\hline $53,916.664 \ldots \ldots$ & 0.654 & 20.7 & 8.0 & 9.3 \\
\hline $53,917.794 \ldots \ldots$ & 0.865 & -2.3 & 8.0 & 12.0 \\
\hline $53,922.691 \ldots \ldots$ & 0.777 & 26.7 & 11.0 & 10.7 \\
\hline $53,923.807 \ldots \ldots$ & 0.985 & -24.3 & 7.0 & 8.0 \\
\hline $53,925.643 \ldots \ldots$ & 0.327 & -3.0 & 8.0 & 7.5 \\
\hline $53,930.644 \ldots \ldots$ & 0.259 & -8.2 & 7.0 & 7.2 \\
\hline $53,932.640 \ldots \ldots$ & 0.631 & 18.5 & 8.0 & 9.5 \\
\hline $53,933.648 \ldots \ldots$ & 0.819 & 19.6 & 12.0 & 11.2 \\
\hline
\end{tabular}

1986). The resolving power of $\sim 100,000$, or $\sim 3 \mathrm{~km} \mathrm{~s}^{-1}$, is much smaller than the profile widths of $\sim 20 \mathrm{~km} \mathrm{~s}^{-1}$, so the observed asymmetries are not compromised by limited spectral resolution. The dispersion is $0.013 \mathrm{m \AA}$ pixel $^{-1}$. Our exposure times are no longer than $2 \mathrm{hr}$, corresponding to $0.8 \%$ of a cycle for $\zeta \mathrm{Gem}, 1.2 \%$ for $\eta \mathrm{Aql}$, and $1.5 \%$ for $\delta$ Cep. Signal-tonoise ratios in the continuum, as estimated from the photon count, range from 140 to 350 .

Figure 1 shows a typical exposure, while Figure 2 shows the $\lambda 6252.57$ profile at a selection of phases to illustrate the changes in shape, strength, and position. The absolute wavelength positions are determined using telluric absorption inside the spectrograph, as described in a previous paper (Gray \& Brown 2006). Barycentric corrections are applied to the wavelength scale using the precepts of Stumpf $(1979,1980)$. The wavelength scale is established with errors of $\sim 25 \mathrm{~m} \mathrm{~s}^{-1}$ (smaller than the symbol size in Fig. 2).

We selected the profiles of six iron lines for analysis: Fe I $\lambda \lambda 6230.74,6232.65,6252.57,6265.16$ and $\mathrm{Fe}$ II $\lambda \lambda 6238.39$, 6247.56. Reasoning that the profile shapes are dominated by the Doppler-shift distributions of pulsation, macroturbulence, and rotation, not the thermal profile of each line, we averaged the six profiles from each exposure. Figure 3 illustrates sets of

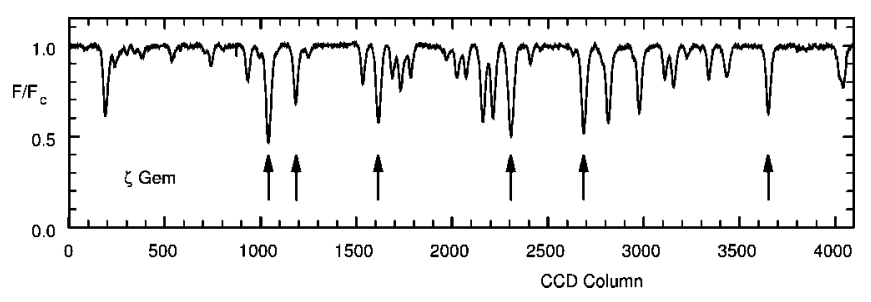

FIG. 1.-Exposure of $\zeta$ Gem, with the six lines we use in our analysis indicated. The label $F / F_{c}$ denotes the flux spectrum normalized to its continuum value. The dispersion is nominally $0.013 \AA$ per column. 

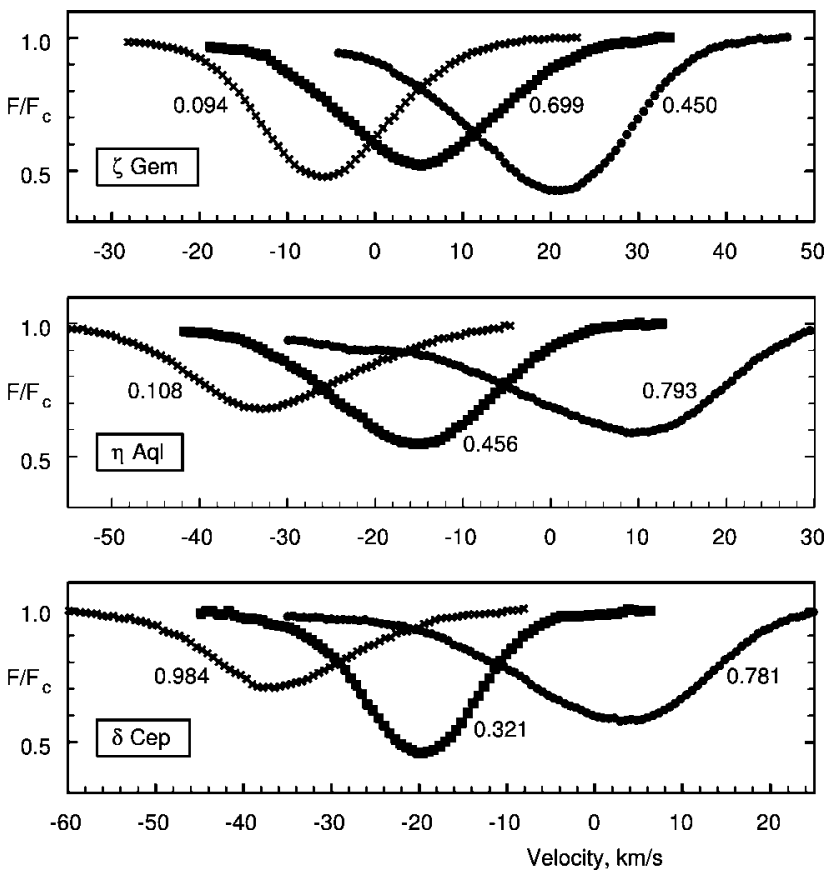

FIG. 2.-Examples of the shapes and positions of Fe I $\lambda 6252.57$ for our three program stars. The ordinate is flux normalized to the continuum; the abscissa is the observed absolute wavelength scale converted to velocity.

profiles from two exposures. The stellar lines change their depths with phase in the cycle, but we normalize them to their central depth in order to focus on the Doppler-shift distribution that shapes them, while playing down the absolute changes in strength. Although there are small differences that are real, they are relatively small compared to the variations shown during the pulsation cycle. An exception is the blending with adjacent weaker lines that occurs in the far wings. We have attempted to manually correct these, but the adequacy of the corrections does not seriously affect the derived pulsation velocities.

\section{THE MODELS}

The models consist of computed Doppler-shift distributions, where disk integration of the line-of-sight components of pulsation, rotation, and an isotropic Gaussian are combined. The Gaussian is meant to simulate the aggregate effects of macroturbulence, velocity gradients within the line-forming layers, unresolved line splitting, and the thermal profile. Its center is displaced to the Doppler shift given by the combined pulsation and rotation velocities appropriate to each point on the stellar disk. There are then three free parameters: (1) the pulsation velocity $V_{\text {puls }}$, (2) the projected rotation velocity $v \sin i$, and (3) the Gaussian dispersion $\zeta$, which we adjust to get agreement with the observations. The pulsation velocity is the fundamental number we seek, but the others are also of some interest (e.g., Kraft 1966; Breitfellner \& Gillet 1993; Bersier \& Burki 1996; Gillet et al. 1999).

The model stellar disk is divided on a sector-radius format, with $0.5^{\circ}$ chosen for the sector step. The radius step is scaled to give dimensions that are comparable to the sector dimension. This results in some 111,000 subareas on the disk. The individual Doppler-shift distributions are weighted by the subarea size and the limb darkening and then summed to get the full Doppler-shift distribution for the whole model star.

Figure 4 shows some basic variations in Doppler-shift distributions (cast as absorption lines) as a function of pulsation

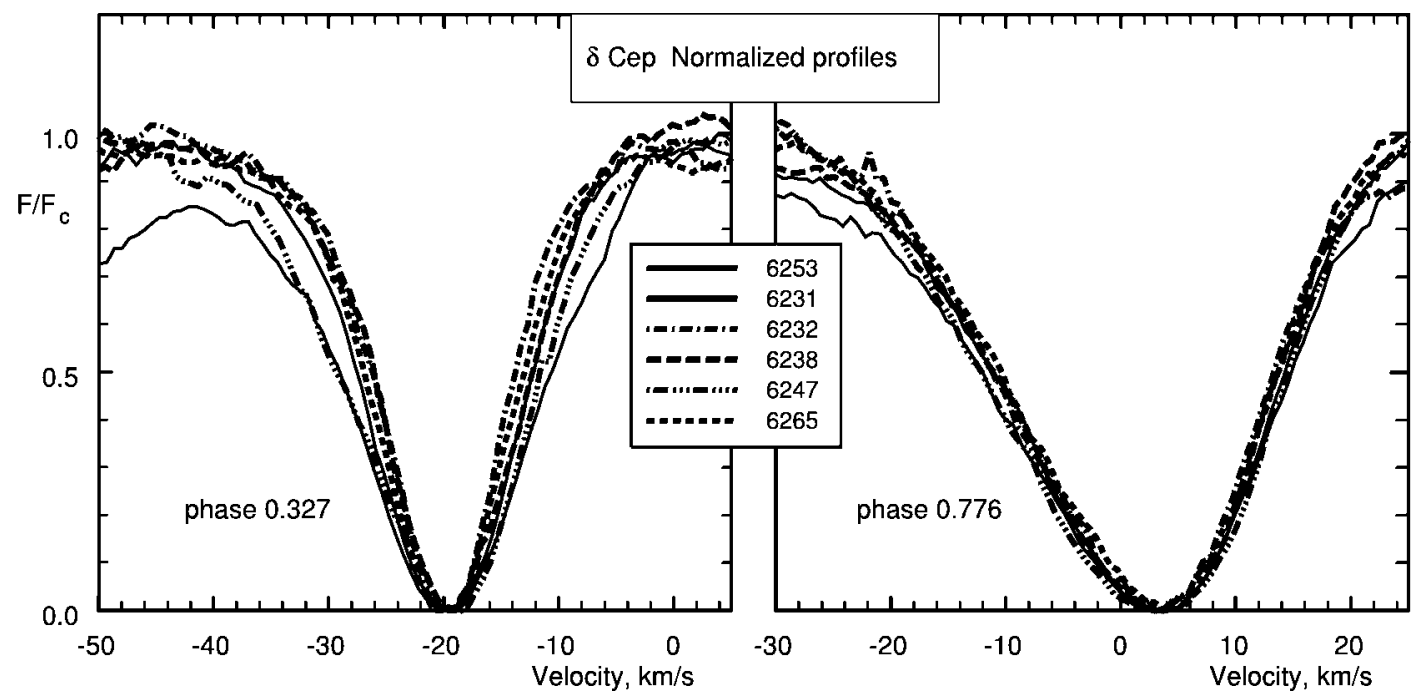

FIg. 3.-Examples of the individual profiles entering the means for two phases of $\delta$ Cep. The set on the left is for a nearly symmetric and narrow-lined phase. The set on the right is for a highly asymmetric case. Deeper profiles (as in Fig. 1) show slightly wider widths, as one generally expects. When the lines have more broadening, as for the set on the right, the agreement improves, showing that all of these lines are experiencing the same Doppler broadening. 


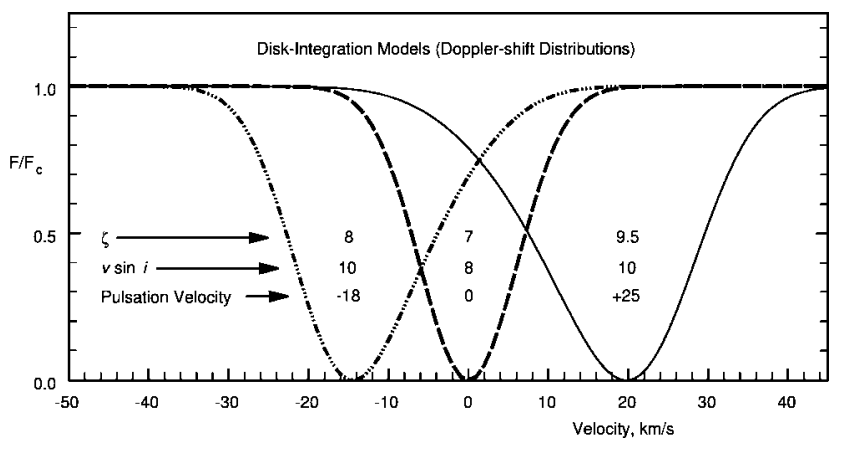

FIG. 4.-Disk-integration models of Doppler-shift distributions. The models mimic both the shape and the shift variations of the observations.

velocity. These variations clearly mimic those seen in Figures 1 and 2. Rotational Doppler shifts play an important role in reproducing the largest asymmetries. Figure 5 illustrates this role. In order to keep the model simple, we have used only rigid rotation. In the real star, rotation may well show complex variations. Typical radius changes of Cepheids are $10 \%$ between maximum and minimum. If the photosphere is weakly coupled to deeper layers, as seems likely, then conservation of angular momentum of photospheric material would imply $\sim 10 \%$ faster equatorial rotation at minimum radius than at maximum radius. Since the pulsation is radial, the change in moment of inertia will vary with the latitude on the star, producing significant differential rotation. Consequently, the physical meaning of rotation in our analysis should be treated with caution, and the derived $v \sin i$ values should be considered characteristic but not definitive. As noted below, the pulsation velocities we derive are nearly independent of uncertainties in $v \sin i$. We also make the approximation of constant limb darkening with phase. We use a limb-darkening coefficient of $\varepsilon=0.7$ in a linear law $I_{\nu} / I_{\nu}^{0}=1-\varepsilon+\varepsilon \cos \theta$, where $\theta$ is the usual limb distance. Our models are not unduly sensitive to this parameter. In most cases, more darkening toward the limb can be compensated by increasing the broadening parameters, while the pulsation velocity one deduces is hardly affected. The worst case is when the asymmetry is largest. Figure 6 illustrates the change for this kind of profile. In this example, the greater limb darkening can be compensated by increasing $v \sin i$ from 8.0 to $8.5 \mathrm{~km} \mathrm{~s}^{-1}$ and decreasing the pulsation velocity from 25.0 to $24.5 \mathrm{~km} \mathrm{~s}^{-1}$, or $-2 \%$.

\section{ANALYSIS}

In order to properly interpret the observations, we must place the stellar profiles on a center-of-mass velocity scale. That is, the space motion or so-called $\gamma$-velocity must be removed. Since we have no way of knowing this when we start, we fall back on the standard condition that the net radius change across a full cycle must be zero. Although Sabbey et al. (1995; see also BöhmVitense et al. 1989) have pointed out how a simple application

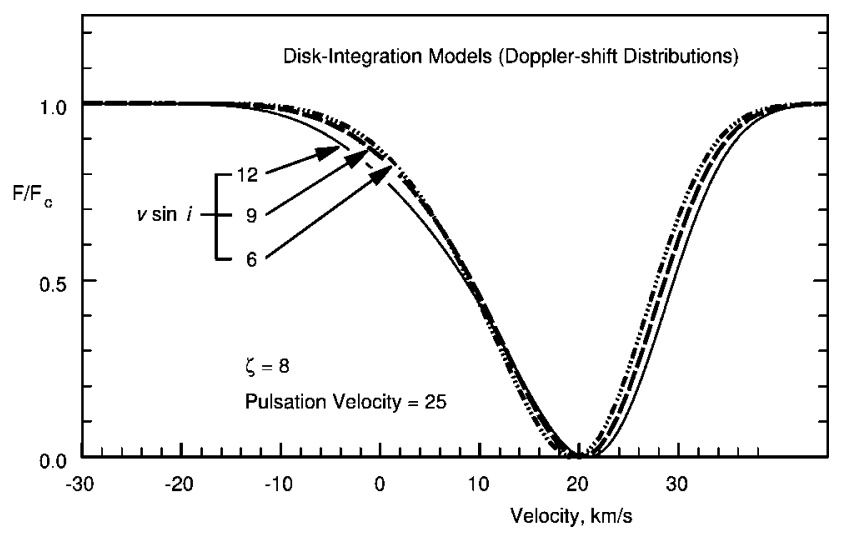

FIG. 5.-Disk-integration models: rotation affects not only the broadening, but also the slope of the less-displaced side of the profile. The range in rotation shown here is larger than that found in our analysis.

of this principle might introduce errors of $\sim 1 \mathrm{~km} \mathrm{~s}^{-1}$ through velocity gradients, this seems unlikely for our stars, as we argue below in $\S 5$. We iterate by starting with a guess at the $\gamma$-velocity based on inspection of the profiles and their asymmetries, then we analyze the observations to get pulsation velocities, integrate the pulsation-velocity curve, and readjust the $\gamma$-velocity to meet the constraint.

Models were matched to the six-line mean profiles for each exposure. Examples are given in Figures 7, 8, and 9. Most observed profiles are reasonably well matched by the models, with deviations rarely larger than the scatter among the six profiles entering the mean. There is a tendency for the top portion of the left wing to be lower in the observations than in the models, which might well result from inadequate corrections for line blending. The models are well constrained by

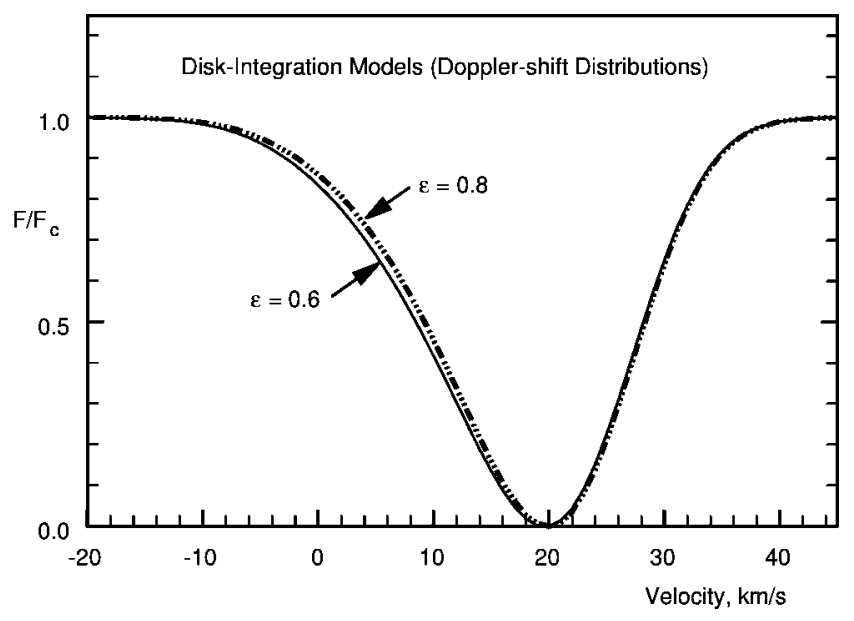

FIG. 6.-Disk-inegration models: altering the limb-darkening coefficient $\varepsilon$ produces a moderate change that can be compensated by changing $v \sin i$ and $\zeta$. The important parameter, the derived pulsation velocity, is changed by only a small amount, in this case $-2 \%$. 


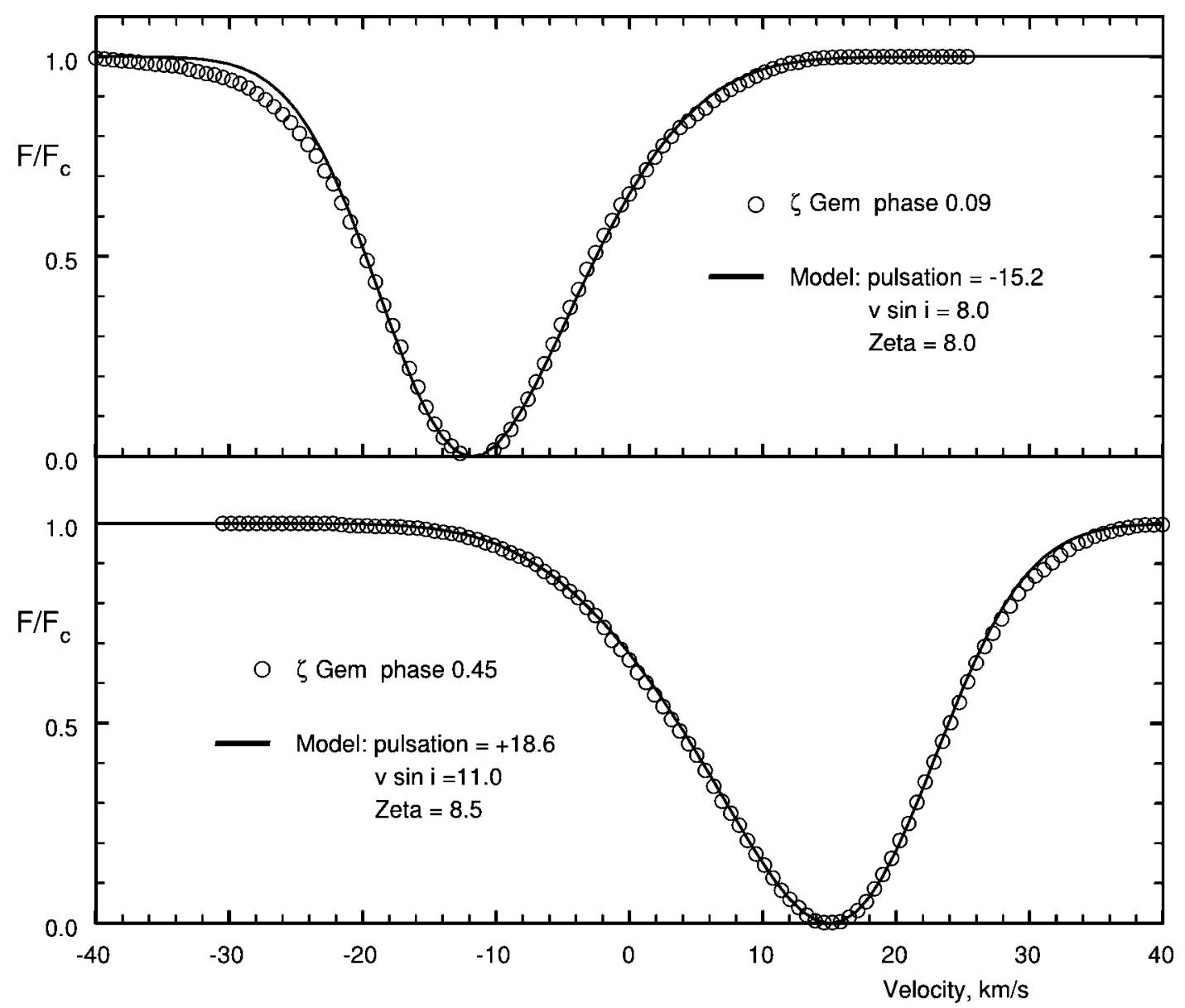

FIG. 7.-Examples of models matched to the observations for $\zeta$ Gem.

the observations. When the pulsation velocity and the asymmetry are large, the amount of asymmetry depends on the rotational broadening, allowing us to fix $v \sin i$ to $\sim 0.5 \mathrm{~km} \mathrm{~s}^{-1}$. Reducing the model's $v$ sin $i$ by this amount increases the magnitude of the deduced pulsation velocity by only $0.1 \mathrm{~km} \mathrm{~s}^{-1}$, or $0.4 \%$. At the opposite extreme, when the pulsation velocity and the asymmetry are small, there is $\sim 2 \mathrm{~km} \mathrm{~s}^{-1}$ cross talk between the macroturbulence dispersion $\zeta$ and the projected rotation rate $v \sin i$, because without the pulsation velocity, both rotation and macroturbulence produce symmetric broadening. Once a choice has been made for a rotation rate, $\zeta$ can be fixed to $\pm 0.3 \mathrm{~km} \mathrm{~s}^{-1}$, or vice versa. The deduced pulsation velocity for this symmetric case is independent of any such trade-off.

The statistical error on individual pulsation velocities arises mainly from the limitation of the signal-to-noise ratio in the observed line profiles. We find from experience that we can see a pulsation-velocity mismatch of $\sim 0.3 \mathrm{~km} \mathrm{~s}^{-1}$ between observed line profiles and the models, and we take this value to be the error in our derived pulsation velocities.

Figures 10, 11, and 12 show the pulsation velocity curves. The line connecting our measurements is a weighted least- squares smoothing of our data. The periods we use are shown on the abscissa labels. They are taken from Kholopov et al. (1998) or Szabados (1991). Because the observations, both ours and those of others, were taken over relatively short time intervals, we do not need a particularly precise period. The measurements from a few other sources are shown in the figures for comparison. Small phase shifts originating in those authors' choice of reference time have been applied to justify them with ours. We arbitrarily scaled the radial velocities of the published data to fit our pulsation velocities. This is equivalent to applying our choice of projection factor (" $p$ " factor) to these data. The agreement is generally rather good.

Integration of the pulsation-velocity curves then gives the radius change. We integrate the absolute value of the pulsation velocity across the full $0-1$ phase interval, and then divide by 2 . This minimizes any residual error in the $\gamma$-velocity, especially for $\eta \mathrm{Aql}$ and $\delta \mathrm{Cep}$, where the expansion and contraction phases are of almost identical duration. The results are $\zeta \mathrm{Gem}$ : $\Delta R=4.88 \times 10^{6} \mathrm{~km} ; \eta$ Aql: $\Delta R=4.59 \times 10^{6} \mathrm{~km}$; and $\delta$ Cep: $\Delta R=3.43 \times 10^{6} \mathrm{~km}$.

Although the power of our method should make these de- 


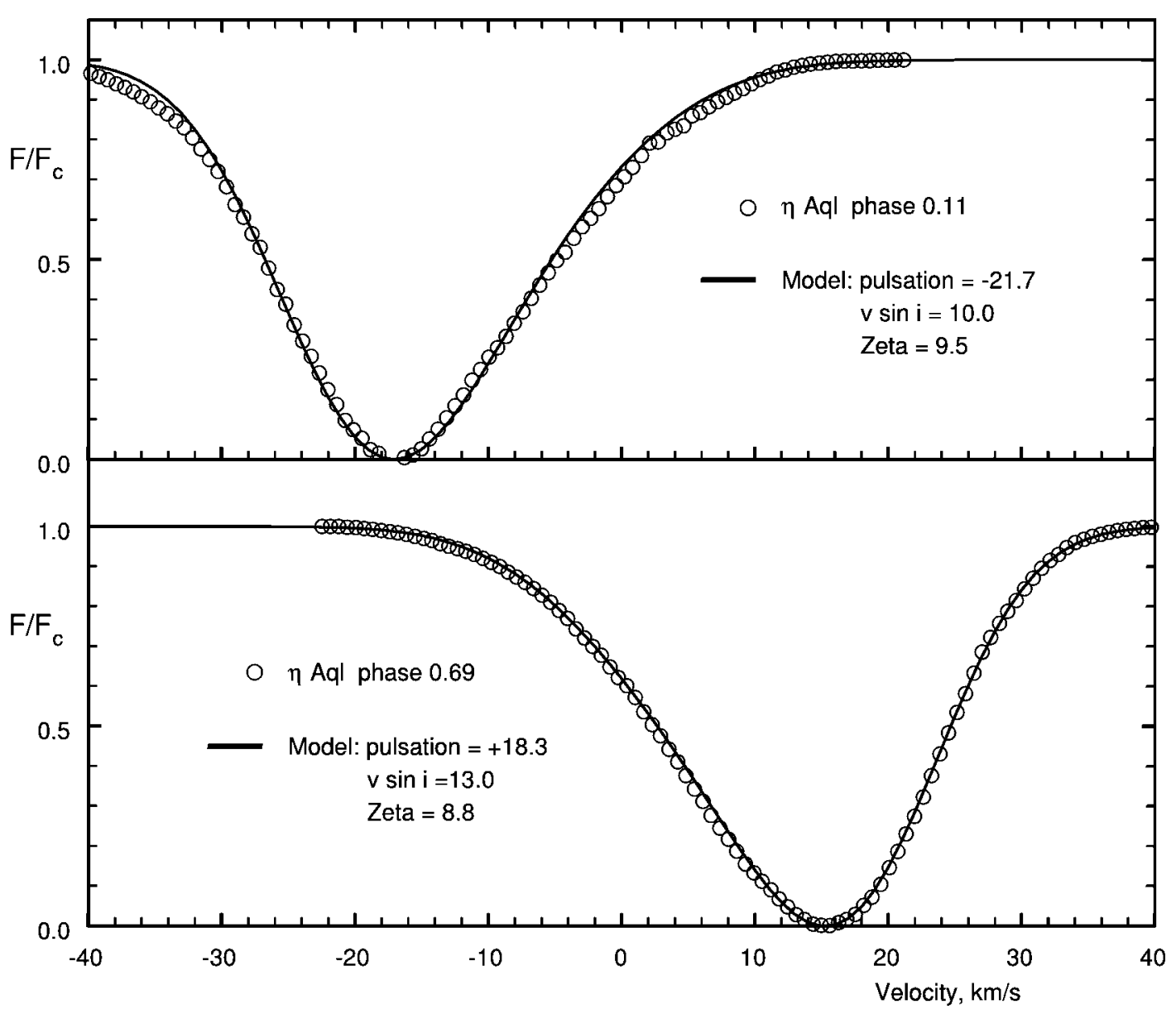

Fig. 8.-Examples of models matched to the observations for $\eta$ Aql.

terminations the best available, their accuracy is limited by our imperfect phase coverage and by other factors, such as limb darkening, that are common to all methods. The estimated $0.3 \mathrm{~km} \mathrm{~s}^{-1}$ error on individual pulsation-velocity measurements implies $\sim 1 \%$ formal error on the radius changes. Another estimate of the precision on $\Delta R$ comes from comparing the values derived from our data alone to a similar solution using all the observations shown in Figures 10, 11, and 12. For $\zeta$ Gem, the difference is $2.1 \%$, for $\eta \mathrm{Aql}$ it is $5.0 \%$, and for $\delta$ Cep there is no difference, or $0.0 \%$. The mean of the three is $2.4 \%$.

\section{DISCUSSION}

By avoiding the ambiguity of the meaning of a radial velocity for asymmetric lines, and by avoiding the application of projection factors, we expected our analysis to show significant differences from previous analyses. Aside from ordinate scaling, our expectation is apparently not fulfilled. Instead, the agreement with previous studies is remarkably close.

There may be a slight divergence for $\eta$ Aql near phase 0.5, although our data are too few to argue the case. However, we note that the mean profile we do have near this phase is slightly asymmetric, the blue wing being deeper than the red wing, even though the pulsation velocity is very near zero. Consequently, a classical radial velocity measurement is likely to give too low a pulsation velocity at this phase. On the other hand, with our model, there is no way to account for the asymmetry at this phase; further investigation is needed.

As a point of comparison, the scaling factors (which amount to empirical $p$-factors) used to fit published data in Figures 10, 11, and 12 are as follows. For $\zeta \mathrm{Gem}$, Nardetto et al. (2006) radial velocities are expanded by 1.48 , compared to their $p$-value of 1.40. We found a factor of 1.42 was appropriate for the Bersier et al. (1994) radial velocities, compared to their choice of $p=1.36$. For $\eta$ Aql, the Butler (1993) measurements were scaled by 1.35 , for Bersier (2002) by 1.33, and for Barnes et al. (2005) by 1.30. For $\delta$ Cep, the stretch factor for the Butler (1993) velocities is 1.36, for Bersier et al. (1994) it is 1.38 , and for Barnes et al. (2005) it is 1.40. Note that since previously published observations scale very well to ours, the deduced $\Delta R$ 's change almost exactly with these scaling factors.

Rotation and its variations during the cycle make important contributions to the shaping of the line profiles. Our geometrical 


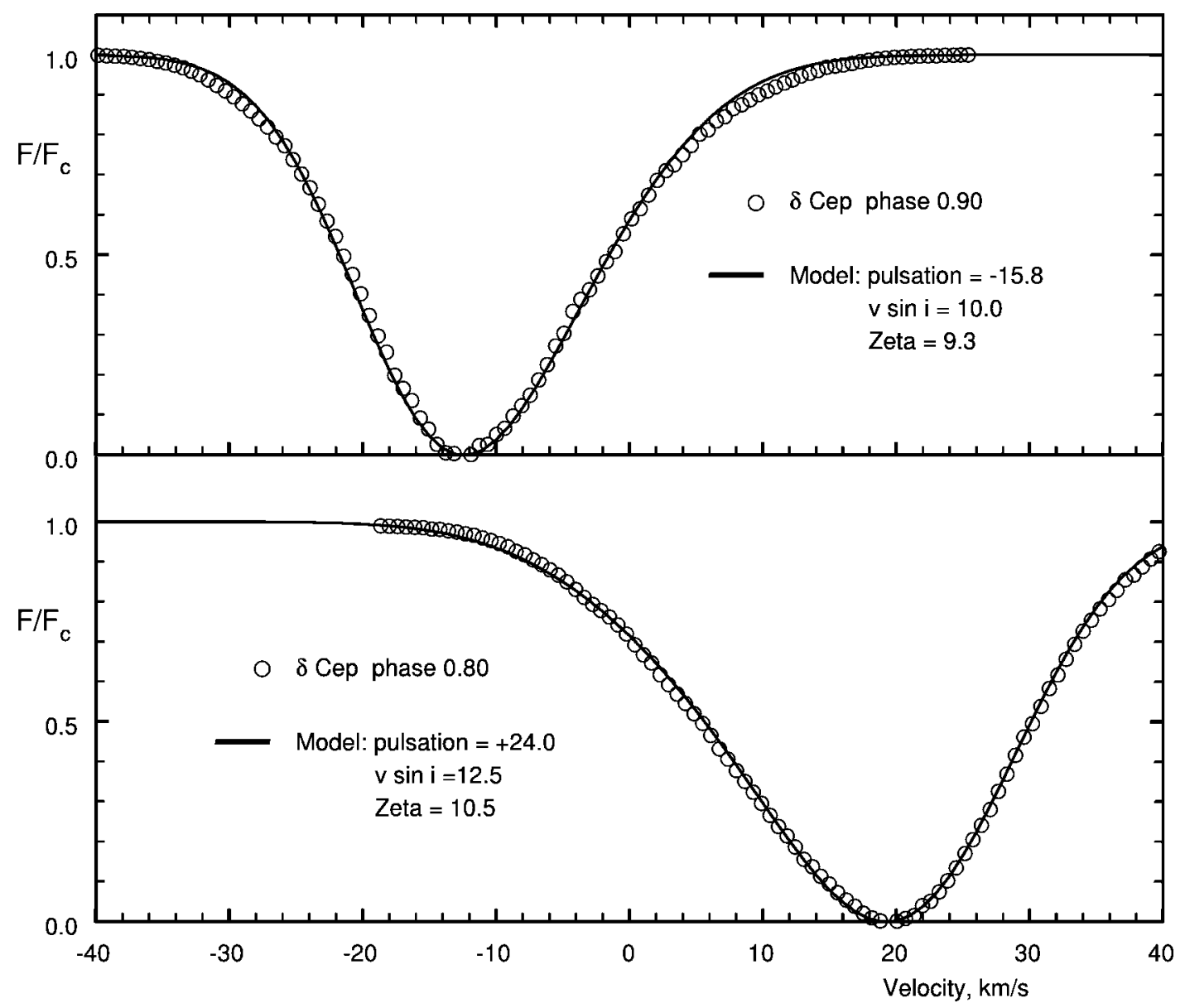

FIG. 9.-Examples of models matched to the observations for $\delta$ Cep.

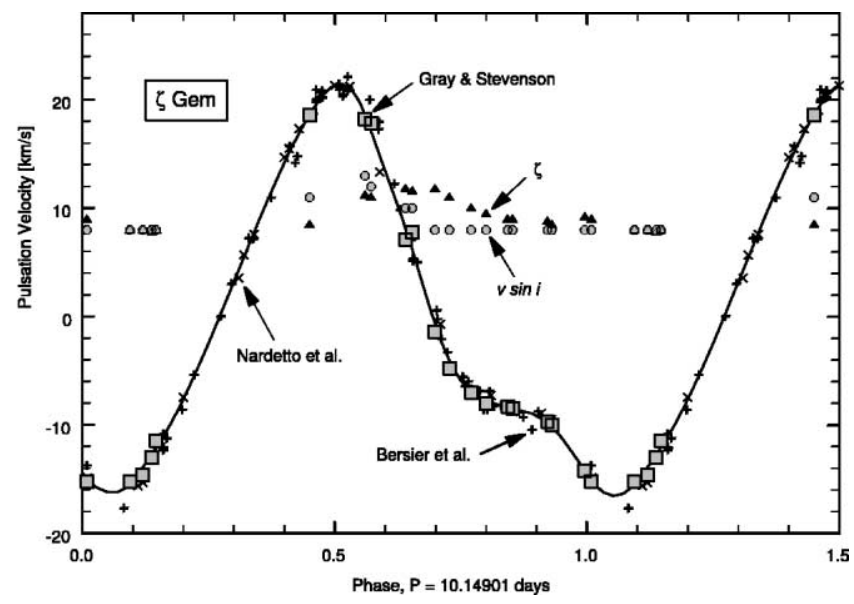

FIG. 10.-Pulsation velocity curve for $\zeta$ Gem. The solid curve is fit through our observations (squares). Macroturbulence dispersion ( $\zeta$; triangles) and rotation rates ( $v \sin i$; gray circles) are also indicated. Crosses $(\times)$ are based on data of Nardetto et al. (2006), and plus signs (+) on data of Bersier et al. (1994).

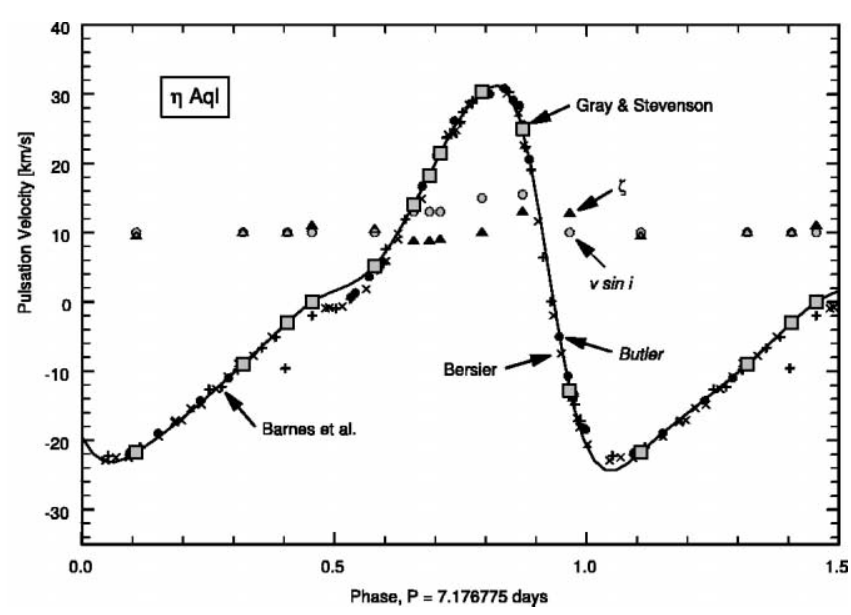

FIG. 11.-Same as Fig. 10, but for $\eta$ Aql. Here filled circles are based on data of Butler (1993), crosses $(x)$ on data of Bersier (2002), and plus signs (+) on data of Barnes et al. (2005). 


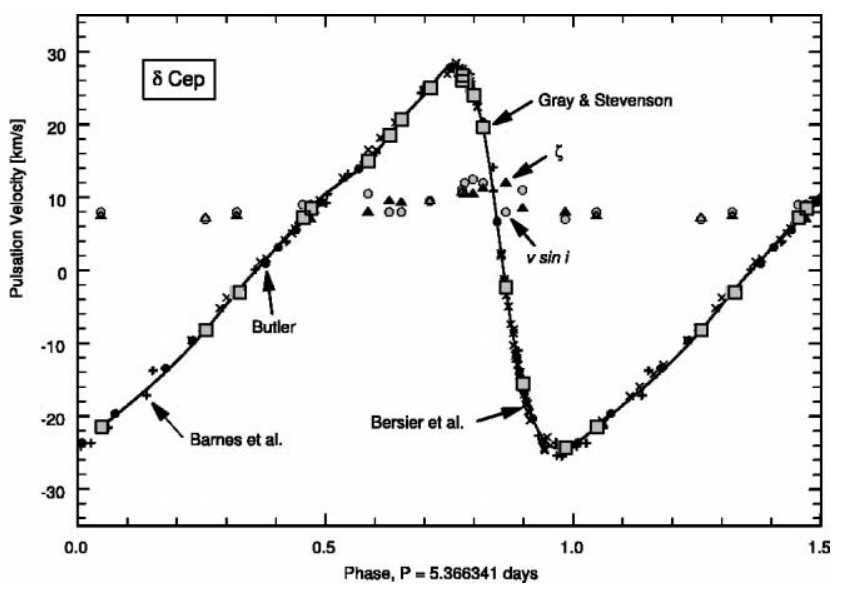

FIG. 12.- Same as Fig. 11, but for $\delta$ Cep. Here crosses $(x)$ are based on data of Bersier et al. ( 1994).

model shows that rotation may have been treated too casually in the past. Even elaborate hydrodynamical models that ignore rotation may well fall short of explaining the observations. The rotation rates we deduce are consistent with those found for similar nonvariable Ib supergiants (Gray \& Toner 1987). We find the rotational broadening is larger at the expected phase (i.e., when the radius shrinks to a minimum), although better phase coverage is needed to make this a definitive statement. However, we do not have a firm handle on how low the rotation may go at maximum radius, since, as we remarked above, rotational broadening can be traded for macroturbulence broadening with considerable impunity. The deduced range in the parameter $v \sin i$ is slightly larger than the simple angular-momentum considerations we noted above.

Naturally, our models have no depth dependence for any of the parameters. Clearly, this is an approximation. However, except for the small contribution of thermal broadening, it is the usual approximation, successfully used in the analysis of the spectral lines of nonvariable stars. The issue to focus on here is therefore the differential pulsation or velocity gradients. The existence of velocity gradients is not in question (e.g., Sanford 1956; Wallerstein et al. 1992; Butler 1993). Rather, we ask whether the velocity gradients produce significant differential shifts in the main portion of the photosphere where our lines are formed. To investigate this, we compared all individual profiles to the six-line means, as in Figure 3, and estimated systematic differences in velocity position. As can be seen in Figure 3, these differences are not large. In most cases, when the differences are plotted as a function of phase, any signal is below the noise level of $0.1-0.2 \mathrm{~km} \mathrm{~s}^{-1}$. In one case, we believe there is a detectable signal with an amplitude of $\sim 0.2 \mathrm{~km} \mathrm{~s}^{-1}$; namely, for our strongest line, Fe I $\lambda 6231$ in $\zeta$ Gem. Based on average line-core depths, this line is $\sim 1.6$ times stronger than the weakest of the six. While this test does not prove that velocity gradients are irrelevant in the formation of our lines, it does show that systematic differences in the pulsation velocity arising from gradients are so small compared to the pulsation amplitude that they can be neglected. This is consistent with our model.

A natural expansion and improvement of our method is to replace the pure-geometry model with a hydrodynamical one that includes rotation while retaining the concepts of fitting the profiles in shape and position and directly yielding the expansion velocity. Likewise, although our model is not applicable to line profiles showing splitting or other complex behavior, the approach we take would still yield the pulsation velocity if more advanced models were able to reproduce the more complicated profiles. The same approach should also be considered for other types of variables.

\section{REFERENCES}

Baade, W. 1926, Astron. Nachr., 228, 359

Barnes, T. G., III, Jeffery, E. J., Montemayor, T. J., \& Skillen, I. 2005, ApJS, 156, 227

Bersier, D. 2002, ApJS, 140, 465

Bersier, D., \& Burki, G. 1996, A\&A, 306, 417

Bersier, D., Burki, G., Mayor, M., \& Duquennoy, A. 1994, A\&AS, 108,25

Böhm-Vitense, E., Garnavich, P., Lawler, M., Mena-Werth, J., Morgan, S., Peterson, E., \& Temple, S. 1989, ApJ, 343, 343

Breitfellner, M. G., \& Gillet, D. 1993, A\&A, 277, 553

Butler, R. P. 1993, ApJ, 415, 323

Fokin, A. B., Gillet, D., \& Breitfellner, M. G. 1996, A\&A, 307, 503

Gillet, D., Fokin, A. B., Breitfellner, M. G., Mazauric, S., \& Nicolas, A. 1999, A\&A, 344, 935

Gray, D. F. 1967, ApJ, 149, 317

- 1986, in IAU Symp. 118, Instrumentation and Research Programmes for Small Telescopes (Dordrecht: Reidel), 401

. 2005, The Observation and Analysis of Stellar Photospheres

(3rd. ed.; Cambridge: Cambridge Univ. Press), 344

Gray, D. F., \& Brown, K. I. T. 2006, PASP, 118, 399
Gray, D. F., \& Toner, C. G. 1987, ApJ, 322, 360

Hindsley, R., \& Bell, R. A. 1986, PASP, 98, 881

Karp, A. H. 1975, ApJ, 201, 641

Kervella, P., Coudé du Foresto, V., Perrin, G., Schöller, M., Traub, W. A., \& Lacasse, M. G. 2001, A\&A, 367, 876

Kholopov, P. N. et al. 1998, Combined General Catalogue of Variable

Stars (ver. 4.1; Moscow: Sternberg Astron. Inst.)

Kiss, L. L., \& Vinkó, J. 2000, MNRAS, 314, 420

Kraft, R. P. 1966, ApJ, 144, 1008

. 1967, in IAU Symp. 28, Aerodynamic Phenomena in Stellar Atmospheres, ed. R. N. Thomas (London: Academic Press), 207

Lane, B. F., Creech-Eakman, M. J., \& Nordgren, T. E. 2002, ApJ, 573,330

Marengo, M., Karovska, M., Sasselov, D. D., Papaliolios, C., Armstrong, J. T., \& Nordgren, T. E. 2003, ApJ, 589, 968

Nardetto, N., Fokin, A., Mourard, D., Mathias, Ph., Kervella, P., \& Bersier, D. 2004, A\&A, 428, 131

Nardetto, N., Mourard, D., Kervella, P., Mathias, Ph., Mérand, A., \& Bersier, D. 2006, A\&A, 453, 309 
Sabbey, C. N., Sasselov, D. D., Fieldus, M. S., Lester, J. B., Venn, K. A., \& Butler, R. P. 1995, ApJ, 446, 250

Sanford, R. F. 1956, ApJ, 123, 201

Sasselov, D., \& Karovska, M. 1994, ApJ, 432, 367

Sasselov, D. D., \& Lester, J. B. 1990, ApJ, 362, 333

Stumpff, P. 1979, A\&A, 78, 229
Stumpff, P. 1980, A\&AS, 41, 1

Szabados, L. 1991, Commun. Konkoly Obs., 96, 123

Wallerstein, G., Jacobsen, T. S., Cottrell, P. L., Clark, M., \& Albrow, M. 1992, MNRAS, 259, 474

Wesselink, A. J. 1946, Bull. Astron. Inst. Netherlands, 10, 91 